\title{
Erratum to: Evaluating next-generation sequencing for direct clinical diagnostics in diarrhoeal disease
}

\author{
K. G. Joensen ${ }^{1,2}$ • A. L. Ø. Engsbro ${ }^{3,4}$ - O. Lukjancenko ${ }^{1}$ - R. S. Kaas $^{1}$ • O. Lund $^{5}$ • \\ H. Westh ${ }^{3,6} \cdot$ F. M. Aarestrup ${ }^{1}$
}

Published online: 9 May 2017

(C) Springer-Verlag Berlin Heidelberg 2017

Erratum to: Eur J Clin Microbiol Infect Dis.

DOI 10.1007/s10096-017-2947-2

Originally published article contains error.

Figures 1, 2 and 3 of the original article were published with missing dots. The correct figures are shown below.

The online version of the original article can be found at http://dx.doi:10.1007/ s10096-017-2947-2

F. M. Aarestrup

fmaa@food.dtu.dk

1 National Food Institute, Division for Epidemiology and Microbial Genomics, Technical University of Denmark, Søltofts Plads,

Building 221, 2800 Kgs. Lyngby, Denmark

2 Department of Microbiology and Infection Control, Statens Serum Institute, Copenhagen, Denmark

3 Department of Clinical Microbiology, Hvidovre University Hospital, Copenhagen, Denmark

4 Department of Medical Gastroenterology, Køge University Hospital, Køge, Denmark

5 Center for Biological Sequence Analysis, Department of System Biology, Technical University of Denmark, Kgs. Lyngby, Denmark

6 Faculty of Health Sciences, University of Copenhagen, Copenhagen, Denmark 

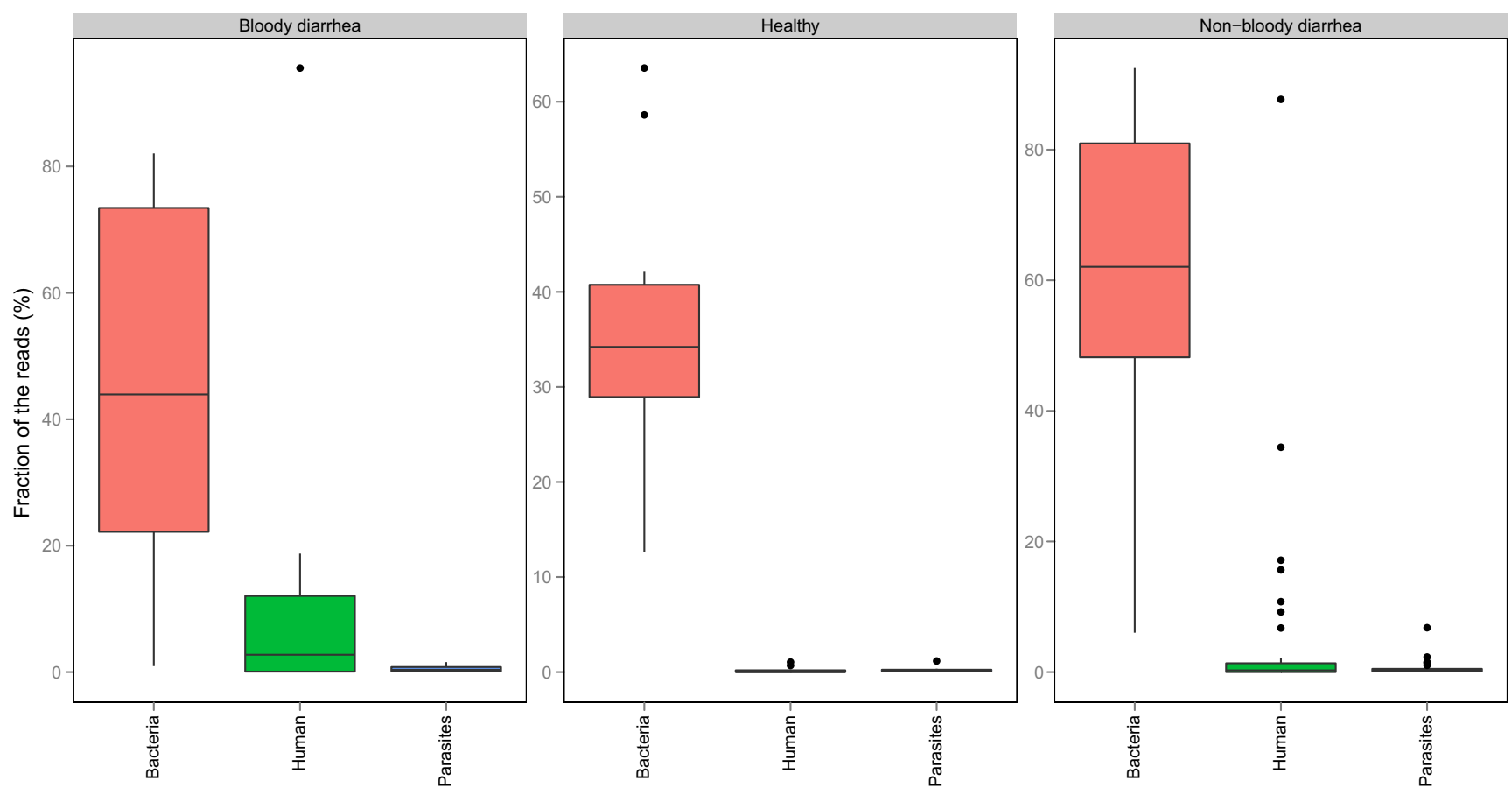

Fig. 1 Abundance of bacterial, parasitic and human DNA among faecal samples. For each group of samples, healthy, patients with bloody diarrhoea and patients with non-bloody diarrhoea (or unknown), the fraction of reads mapping to bacteria, parasites and human reference genomes is shown. The abundance is normalised according to the total number of reads in each specific sample 

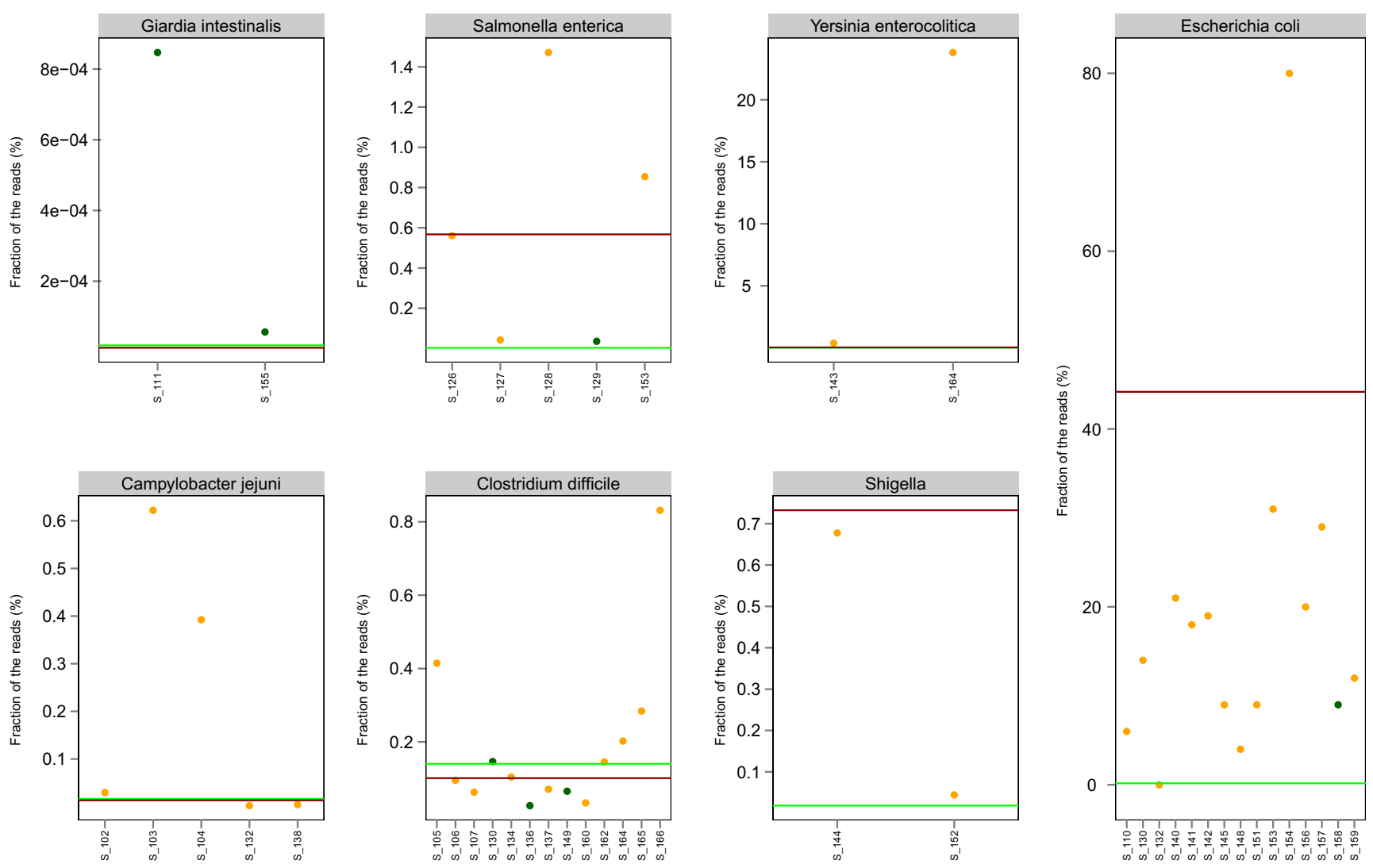

Fig. 2 Relative abundance of pathogens in samples negative by conventional diagnostics. For samples that were either negative or virus-positive by conventional diagnostics, the fraction of reads mapping to each pathogen (Giardia, Salmonella, Y. enterocolitica, E. coli, C. jejuni, C. difficile and Shigella) was plotted. The orange dots

indicate the presence of pathogen-specific virulence genes, while the green dots indicate the absence. The upper fence $\left(\mathrm{Q}_{3}+1.5 \times \mathrm{IQR}\right)$ of the relative abundance for healthy controls and for the diarrhoea samples where the particular pathogen was not detected by conventional methods are shown 

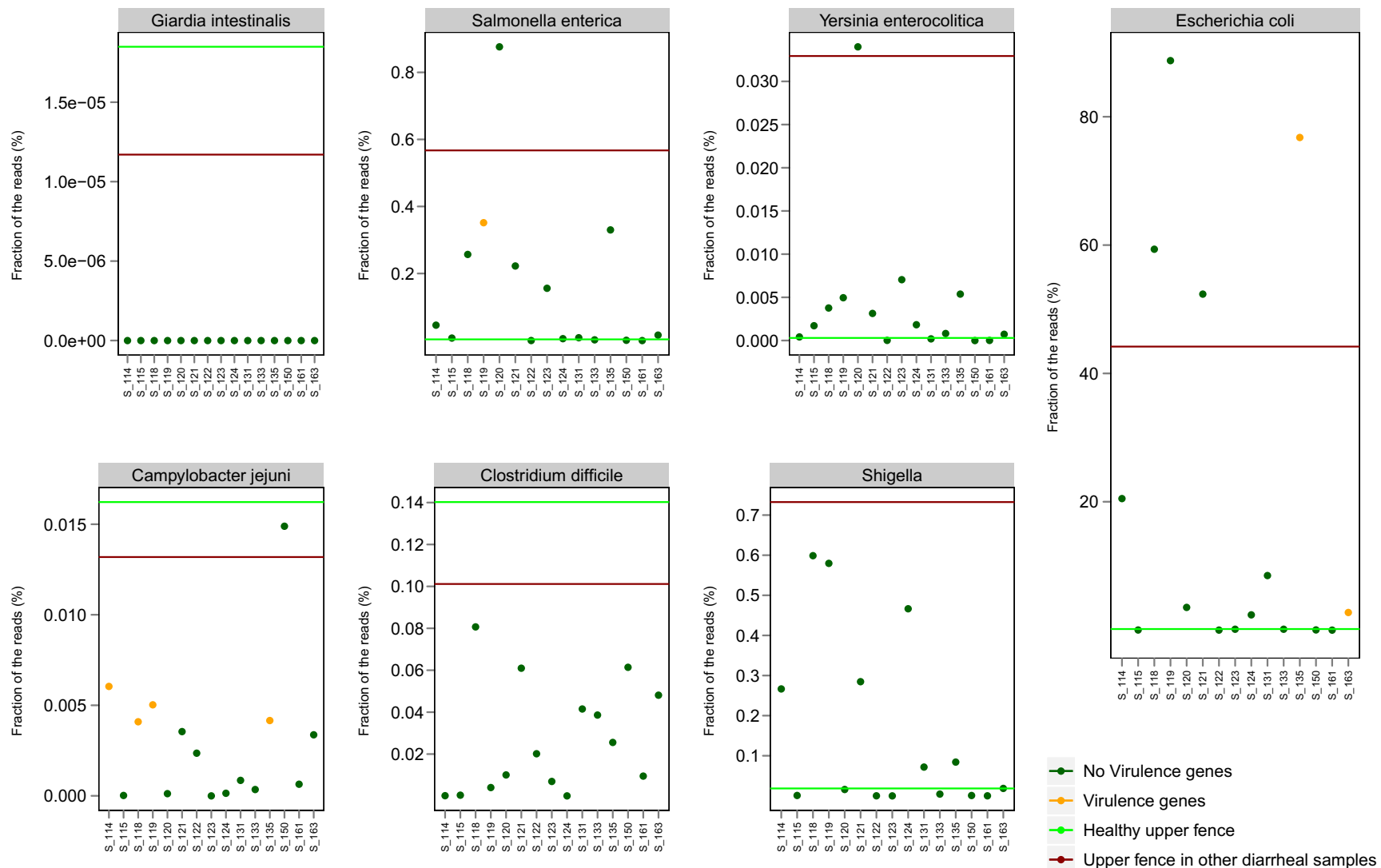

$\rightarrow$ Upper fence in other diarrheal samples

Fig. 3 Relative abundance of pathogens in samples positive by conventional diagnostics. For each pathogen (Giardia, Salmonella, Y. enterocolitica, E. coli, C. jejuni, C. difficile and Shigella), the fraction of reads mapping to the pathogen is plotted for all samples positive by conventional diagnostic methods. The orange dots indicate the presence

of pathogen-specific virulence genes as determined by NGS analysis, while the green dots indicate the absence. The upper fence $\left(\mathrm{Q}_{3}+\right.$ $1.5 \times \mathrm{IQR})$ of the relative abundance for the healthy controls and for the diarrhoea samples where the particular pathogen was not detected by conventional methods are shown 